\title{
Effects of encoding instructions and retrieval cuing on recall in Korsakoff patients
}

\author{
JOHN MCDOWALL \\ Porirua Hospital, Porirua, New Zealand, \\ and Victoria University of Wellington, New Zealand
}

\begin{abstract}
The relationship between the depth of encoding a word and its subsequent recall, either cued or noncued, was investigated in this study. In Experiment 1, Korsakoff subjects and alcoholic controls were shown a categorized word list under one of three different encoding instructions: (1) nonsemantic, that is, detecting the presence or absence of the letter " $e$ " in each word, (2) semantic, that is, assigning words to their correct taxonomic category, and (3) no encoding instructions. Semantic encoding instructions resulted in higher recall for both diagnostic groups than the other instructions. In Experiment 2, subjects were again assigned to one of the three encoding instructions as in Experiment 1, but all groups received cues (category labels) at the time of recall. Cuing increased recall for all but the group receiving instructions to encode nonsemantically. Experiment 3 was a replication of the previous experiments. The results indicated that Korsakoff subjects were capable of encoding semantically without specific instructions to do so but were impaired in the ability to generate retrieval cues at the time of recall.
\end{abstract}

The clinical symptoms of Korsakoff's syndrome have been carefully described by a number of authors (see Talland, 1965; Victor \& Yakovlev, 1955, for reviews). While there is considerable variation in the nature of the illness between individuals, it is generally agreed that it takes the following course: The patient is admitted to a hospital showing signs of acute confusion and agitation. Typically, he is disoriented in time and place and may display evidence of ataxia and extraocular movements. A history of severe alcohol abuse is usually noted. The acute confusional state may last for several days or weeks, after which the patient usually becomes correctly oriented and, in general, is able to perceive his surroundings correctly. He can understand sentences and can usually engage in rational conversation.

Closer examination of the patient at this time reveals a profound amnesia in which the memory for recent events is severely impaired. The patient can typically recall events from the remote past correctly, but in some cases these are recalled out of their correct temporal sequence. Immediate memory, as measured by digit span tests, is not impaired (Talland, 1959; Victor, Herman, \& White, 1959), and the IQ usually remains

The author would like to thank the Wellington Hospital Board for the use of their facilities during the preparation of this manuscript. The valuable encouragement and assistance of Murray J. White, K. G. White, and S. Niemiec is gratefully acknowledged. Requests for reprints should be sent to John McDowall, Psychology Department, Porirua Hospital, Private Bag, Porirua, New Zealand. within the normal range. In addition to a retrograde amnesia, the ability to acquire new information is severely impaired (anterograde amnesia). The patient is often unable to recall the name of the hospital or ward he resides in and appears unable to learn the names of the staff with whom he regularly deals.

While a great deal of clinical and anecdotal evidence exists on the nature of the memory deficit (see Meissner, 1968; Talland, 1965, for reviews), it has only recently been investigated through the use of information processing techniques. Most emphasis has been placed on the nature of the anterograde amnesia, and different workers have debated as to whether it is due to imperfect initial encoding, impaired retrieval abilities, or a combination of these and other factors.

In current memory research, there is considerable evidence to suggest that stimuli may be encoded in different ways within the memory system. A word may be encoded in terms of its physical features (e.g., as to whether or not it contains the letter " $\mathrm{m}$ "), or it may be encoded in terms of its phonemic features (e.g., as to whether it rhymes with the word "cat"). A word may also be encoded semantically, that is, in terms of its meaning (e.g., as to whether the word refers to a type of animal). Cermak (1972), Craik and Lockhart (1972), and Craik and Tulving (1975) have hypothesized that the persistence of the memory trace is a function of the depth of analysis performed on the stimuli with deeper (semantic) levels of encoding being associated with longer lasting memory traces (and thus higher recall performances), as compared with nonsemantic levels of encoding that result in rapidly decaying memory traces. 
There is now a considerable body of evidence that shows that, normally, semantic encoding results in higher performance levels than physical or phonemic encoding for both recall and recognition tasks (Craik \& Tulving, 1975; Fisher \& Craik, 1977; Hyde, 1973; Hyde \& Jenkins, 1973; Till \& Jenkins, 1973).

Cermak and Butters (1973) hypothesized that Korsakoff patients suffer from a basic impairment in the ability to encode stimuli, particularly along semantic dimensions, relying rather on physical and phonemic levels of encoding. The initial evidence for this view was provided by Cermak and Butters (1972), who had Korsakoff subjects listen to a list of eight words containing two words from each of four taxonomic categories. The subjects were asked to recall the words in any order (free recall). A second list of eight words belonging to the same four categories was administered to the same subjects, who were then told the category names before the list presentation and were also informed that they would be required to recall the words category by category as directed by the examiner (cued recall). Words were presented orally at a rate of 1 word/sec. Korsakoff subjects recalled significantly fewer words under the cued condition than under the free condition, whereas a control group of alcoholics showed improved recall under the cued condition. The authors concluded that, since the Korsakoff subjects did not benefit from the provision of semantic cues (category labels), they were not employing semantic encoding strategies but were probably relying on auditory encoding.

There are two main problems with the conclusions of this study. First, the design of the experiment may have inadvertently maximized proactive interference (PI), in that List 2 consisted of words taken from the same taxonomic categories as those used in List 1. The poor performance of the Korsakoff subjects on List 2 may have reflected an excessive susceptibility to PI. This effect has been confirmed in a number of independent experiments (Warrington \& Weiskrantz, 1970, 1974). Second, there is evidence indicating that Korsakoff subjects are impaired in the rate at which they can encode information. Oscar-Berman, Goodglass, and Cherlow (1973) used a visual backward masking paradigm to examine information processing times in Korsakoff subjects and non-Korsakoff controls and found that Korsakoff subjects had abnormally high visual processing times. The 1-word/sec presentation rate used in the Cermak and Butters (1972) study may have been inadequate to allow semantic encoding. Providing category cues may have interfered with rote recall.

Cermak, Butters, and Gerrein (1973) carried out four experiments to investigate further the semantic encoding ability of Korsakoff subjects. One experiment was an attempt to reconcile the findings of Cermak and Butters (1972) that Korsakoffs were unable to benefit from category cuing with those of an earlier experiment by Warrington and Weiskrantz (1971), who found that category cuing increased recall compared with a noncued condition. The results of this experiment showed that, while providing category labels at presentation and recall time did not facilitate immediate recall, it did facilitate retention after a 1 -min delay. The authors did not attempt an explanation of this interaction, but they did conclude that Korsakoff subjects were capable of encoding words by their semantic properties "to some extent" (Cermak et al., 1973 , p. 87). A second experiment involved reading a list of words to subjects and then providing cues for the recall of certain words within the list. The cues used were either acoustic (i.e., a rhyme of the to-beremembered word) or semantic (i.e., the category name of which the to-be-remembered word was a member). The rationale for this design was provided earlier by Bregman (1968), who showed that semantic cuing was of value only if the stimuli had been encoded at this level. If the subjects had only encoded stimuli at an acoustic level, then only acoustic cuing should aid recall. In the second experiment, the effects of both acoustic and semantic cues were assessed by reading subjects a list of words and then providing a cue to a word that had appeared in the list one, two, three, or six items prior to the cue item. Before each list presentation, subjects were informed as to which type of cue would be provided. A group of alcoholic patients served as a control group. The authors concluded that "except for this slightly faster rate of decay in the effectiveness of the category cues, the Korsakoff and alcoholic patients benefited equally from both acoustic and semantic cues" (Cermak et al., 1973, p. 88).

An examination of the results of this experiment raises doubts as to the validity of this conclusion and, in a review article (Cermak \& Butters, 1973), a modification of the earlier interpretation is presented. Thus, "the rhyming and category cues were equally effective in both groups when the to-be-recalled item and its cue were separated by only one or two intervening items, but with longer delays (three or six items between test item and cue) between presentation and cued recall the category cue was more effective among the controls... . The sharp decline in the facilitative effects of the category cue in the Korsakoff patients seemed to indicate a deficiency in the original semantic encoding of the tobe-recalled words" (Cermak \& Butters, 1973, p. 1121).

Unfortunately, this modified conclusion is also at odds with the original finding, in which the analysis of variance indicated that the Korsakoff patients recalled significantly fewer items than the controls only after a three-item delay between test item and category cue. In other words, both the Korsakoff patients and the controls recalled equally effectively after one, two, and 
six intervening items between test item and category cue, a finding that would be difficult to interpret in terms of a semantic encoding deficit.

The third experiment in this study (Cermak et al., 1973) consisted of presenting subjects with a series of words and measuring recall after providing them with associative cues (i.e., words that were semantically associated with the to-be-remembered words). The results showed that Korsakoff subjects were capable of taking advantage of such cuing, indicating that they were encoding words along semantic dimensions. This conclusion was reiterated in a review article by Cermak and Butters (1973), who stated that Korsakoff subjects "may be capable of encoding semantically but may prefer to rely on an acoustic or associative level of encoding" (p. 1123).

Cermak, Butters, and Moreines (1974) used the Wickens (1970) "release from PI" paradigm to examine the semantic encoding abilities of Korsakoff subjects. This technique is a modification of the Peterson and Peterson (1959) procedure. Subjects are presented with a series of trials (usually four) in which to-beremembered words are presented, and the words fall into a similar class or category (e.g., all animal names). Recall is measured at the end of each trial and is typically found to decrease over trials due to the hypothesized build-up of PI. On Trial 5, words are presented from an original class or category, and the amount of PI "release" is measured by the increase in performance that results from the introduction of the material from the new category.

Beginning with the hypothesis that the Korsakoff subjects displayed an inability to semantically encode stimuli, Cermak et al. (1974) predicted that the amount of PI release shown would vary with the encoding requirements of the material presented. Their results showed that when the shift in to-be-remembered material was of an alphanumeric nature (consonant trigrams presented on the first four trials, shifting to a digit triad on Trial 5), both Korsakoff subjects and controls displayed a PI release. But, when the shift was of words taken from one taxonomic category on Trials $1-4$ to words from a different taxonomic category on Trial 5, Korsakoff subjects showed no gain in performance as opposed to the control group. The authors concluded that the Korsakoff patients were unable to encode the words on the basis of their semantic properties and thus gained no benefit from a change in taxonomic category.

While it is possible to view this finding as the result of a failure to semantically encode, it could also be viewed as reflecting a retrieval difficulty. In other words, Korsakoff subjects may have semantically encoded the new class of material but failed to gain an increase in performance because of a failure to generate appropriate retrieval cues. This hypothesis was investigated by Wood and Kinsbourne (Note 1), who obtained a delayed release of PI by continuing to provide the new class of words on Trials 6 and 7. They argued that the delayed release of $P I$ implicated a retrieval failure rather than an encoding impairment.

Other investigators have also viewed the Korsakoff memory deficit as a retrieval difficulty. Jaffe and Katz (1975) examined the relative effects of providing category label cues at presentation (precuing) and at recall times (postcuing). This experiment used only one subject. Presenting a 25 -word list of 5 words falling into five taxonomic categories, Jaffe and Katz tested the subject on a free recall test followed by a recognition test. The list was presented under four different cuing conditions: (1) no cues, (2) postcuing only (i.e.,providing the subject with the category names at recall time), (3) precuing only (i.e., instructing the subject at the time of presentation as to the different categories involved in the word list), and (4) both pre- and postcuing. The results supported a hypothesis that involved a deficit in both encoding and retrieval, in that there was no postcuing advantage unless precues had been provided. Precues alone also had no beneficial effect. The results were compatible with the encoding specificity hypothesis (Tulving \& Thomson, 1973), which states that "specific encoding operations performed on what is perceived determines what is stored and what is stored determines what retrieval cues are effective in providing access to what is stored" (p. 369).

In another study that implicates a retrieval difficulty in Korsakoff subjects, Wood and Kinsbourne (Note 1) presented subjects with lists of three words, words in each list being taken from a single category (e.g., red, blue, green). Three such lists were presented, each being separated from the other by $30 \mathrm{sec}$ of digit subtraction. Precued subjects were told the name of the category labels at the time of presentation. In the postcuing condition, subjects were told the category name at the time of recall and asked to name the words in the list. This study showed that precuing did not benefit recall but postcuing did. The authors argued that the failure to find any benefit from precuing would not be predicted from a semantic encoding deficit hypothesis, since an encoding deficit should have been at least partly overcome by precuing. This conclusion is only valid, however, if the assumption is made that providing Korsakoff subjects with the appropriate category name at the time of presentation somehow cues them to process the words along semantic dimensions. The failure to find any precuing advantage in relation to a non-precued condition may have been due to the inadequacy of the precue to assist semantic encoding.

One of the difficulties with many of the studies discussed above is the ambiguous nature of the encoding strategy adopted by the subjects during the list presentation. In some cases subjects are given instructions to remember, and the level of encoding is inferred from the nature of the recall. In other studies subjects are 
presented with word lists and cued by category labels at the time of presentation. The interpretation of the recall performance under this procedure requires the assumption that providing subjects with the appropriate category name at presentation time cues subjects to process the words along semantic dimensions. There is, however, no independent way of showing that such semantic encoding has occurred. What is required is to bring the encoding strategies of the subject under experimental control. In this way the effects of encoding strategies on recall and the effects of retrieval cuing under known encoding strategies can be assessed.

The present experiments bring the encoding strategies of the subjects under control by varying the instructions given to the subjects prior to the presentation of word lists. Consider the procedure in which Korsakoff subjects are presented with the word "cat" and are instructed to place it under its correct superordinate label (i.e., animal). Correct allocation would require that subjects encode the words along semantic dimensions. The ability of subjects to perform this task accurately provides a direct check on whether semantic encoding occurred or not. Similarly, asking subjects to detect the presence or absence of the letter " $\mathrm{e}$ " in each word presented allows the argument that the word has been encoded along nonsemantic levels. Experiment 1 used this procedure to examine the relative effects of three different encoding instructions on recall: (1) semantic (i.e., allocating words to their correct category labels), (2) nonsemantic (i.e., detecting the presence or absence of the letter " $\mathrm{e}$ " in each word), and (3) no instructions (i.e., subjects were asked to remember the words). Experiment 2 examined the effects of the three encoding instructions in the presence of semantic cues provided at recall time. Experiment 3 was a replication of Experiments 1 and 2.

\section{EXPERIMENT 1}

This experiment examined the hypothesis summarized by Cermak and Butters (1973) that Korsakoff subjects are impaired in the ability to encode stimuli along semantic dimensions. There are two related aspects to this hypothesis. The first is that Korsakoff subjects are capable of encoding stimuli along semantic dimensions when given specific instructions to do so. Second, without such instructions, Korsakoff subjects encode stimuli on nonsemantic levels. Experiment 1 compared the recall performance of a group of Korsakoff subjects receiving no explicit encoding instructions with a group receiving instructions to encode stimuli along nonsemantic dimensions and another group engaged in a task designed to maximize semantic encoding. If Korsakoff subjects typically encode words along nonsemantic levels in the absence of any explicit encoding instructions, then their recall performance should be similar to a group of Korsakoff subjects instructed to encode stimuli on a nonsemantic level.

\section{Method}

Subjects. Thirty-six male patients were drawn from the population of a large psychiatric hospital. The experimental group consisted of 18 subjects diagnosed as Korsakoff patients with an alcoholic history. The remaining 18 subjects served as a control group and were diagnosed as alcoholic with no known evidence of brain damage or memory impairment. Thus, both groups had a history of severe alcohol abuse. The mean age of the Korsakoff group was 55.33 years (range: 45-66 years), and the mean IQ (Wechsler Adult Intelligence Scale, WAIS, verbal IQ) was 96.38 (range: 89-104). The mean age of the alcoholic group was 55.0 years (range: $39-67$ years), and the mean IQ was 93.55 (range: 89-108). Korsakoff subjects were not selected if they displayed any evidence of dementia.

Subjects within each of the two diagnostic groups were randomly assigned to one of three groups, classified according to encoding instructions: (1) nonsemantic encoding instructions (NS), (2) semantic encoding instructions (S), (3) no explicit encoding instructions $(\mathrm{N})$

Stimuli and Procedure. Stimulus materials were 20 $20 \times 12.5 \mathrm{~cm}$ white cards with one word printed on each card. The 20 words consisted of five nouns from each of four taxonomic categories (kitchen utensils, food, clothing, and animals). Subjects were tested individually and sat facing the experimenter across a table upon which the cards were presented one at a time. Subjects in the NS group were instructed to detect the presence or absence of the letter " $e$ " in each word and to indicate their decision to the experimenter by pointing to one of two cards on which were printed the words "yes" and "no." All subjects in this group were told that a recall test was to be given afterward. Subjects in Group N were instructed to remember the stimulus words in preparation for a recall test. Subjects in Group $S$ were instructed to examine each word as it was presented and to decide to which taxonomic category it belong. The four superordinate category names were printed on four cards and were placed on the table in front of the subject, who was required to point to the appropriate label upon the presentation of the stimulus. Subjects in this group were also told of the recall test that was to follow. Each word was presented for $4 \mathrm{sec}$ and, at the end of the first presentation, a second presentation was given. At the end of this, subjects were given the recall test. Cards were presented in a random order with the constraint that no two members of the same taxonomic category be presented consecutively.

\section{Results and Discussion}

The mean percentage correct recall scores for each of the two diagnostic groups under the three encoding instructions are shown in Figure 1. The main effect of diagnostic group was significant $[F(1,30)=55.04$, $p<.001]$, as was the effect of encoding instruction $[F(2,30)=16.65, p<.001]$ and the interaction between diagnostic group and encoding instruction $[F(2,30)=3.44, p<.05]$.

Multiple comparisons of the mean recall scores for the Korsakoff subjects under the three encoding instructions showed differences between NS and $\mathrm{S}$ instructions $[F(1,30)=16.12, p<.001]$ and between $N$ and $S$ instructions $[F(1,30)=8.90, p<.01]$. There was no difference between the means for NS and $\mathrm{N}$ instructions $[\mathrm{F}(1,30)=1.06, \mathrm{p}>.05]$.

For the alcoholic subjects, there were significant differences between the means for NS and $S$ instructions $[F(2,30)=16.13, p<.001]$ and between $N S$ and $N$ instructions $[F(2,30)=18.02, p<.001]$. There was no difference between the means for $\mathrm{N}$ and $\mathrm{S}$ instructions 


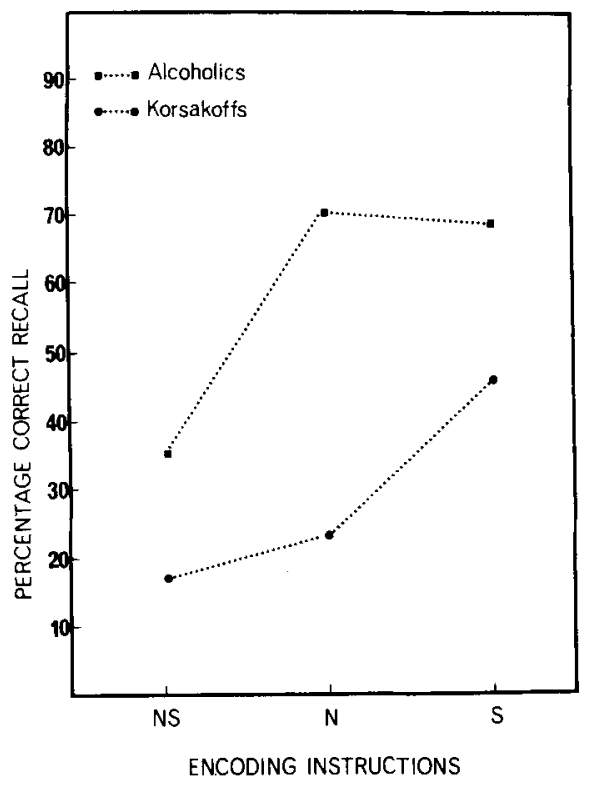

Figure 1. Mean percentage correct recall for Korsakoff and alcoholic groups as a function of encoding instructions (NS = nonsemantic instructions, $N=$ no instructions, $S=$ semantic instructions).

$(\mathrm{F}<1)$. Between-group comparisons showed the alcoholic group to have higher recall scores than the Korsakoff group for NS instructions $[\mathrm{F}(2,30)=10.32$, $\mathrm{p}<.01]$, for $\mathrm{N}$ instructions $[\mathrm{F}(2,30)=41.29, \mathrm{p}<.001]$, and for $S$ instructions $[F(2,30)=10.32, p<.01]$.

The results of this experiment show that when Korsakoff subjects are given no specific encoding instructions, their recall performance is the same as that of a group of Korsakoff subjects instructed to encode words along nonsemantic levels. In turn, the absence of specific encoding instructions results in a lower recall performance when compared to a group of Korsakoff subjects instructed to encode stimuli semantically. For the alcoholic group, instructions to encode words along their semantic dimensions resulted in a higher recall than that obtained by the group instructed to encode nonsemantically. Recall performance in the absence of explicit encoding instructions was equal to the performance obtained by the group instructed to encode semantically.

While the results obtained from the Korsakoff subjects provide support for Cermak and Butters' (1973) theory that Korsakoff subjects do not encode stimuli semantically unless instructed to do so, the findings could also be viewed as the result of a difficulty in retrieving information. In other words, the Korsakoff subjects who received no explicit encoding instructions may have semantically encoded the words adequately but failed to increase their recall scores above that of the group receiving instructions to encode nonsemantically because of a failure to generate their own retrieval cues. This argument could explain the higher recall performance of the group receiving instructions to encode semantically, if presenting superordinate labels during the learning phase provided subjects with direct access to retrieval cues. In other words, the subjects receiving instructions to encode stimuli semantically were also directly provided with cues that may have aided them at recall. If Korsakoff subjects in the absence of explicit encoding instructions do encode stimuli along semantic dimensions, then providing category label cues at the time of recall should facilitate performance. On the other hand, if they do not encode semantically in the absence of explicit instructions to do so, then semantic cues presented at recall should be relatively ineffectual. Experiment 2 used the same encoding instructions as in Experiment 1 and provided category label cues at the time of recall.

\section{EXPERIMENT 2}

\section{Method}

Subjects. Thirty-six male patients who had not served as subjects in Experiment 1 were used in the present experiment. Eighteen subjects were diagnosed as alcoholic Korsak off patients, and 18 subjects diagnosed as alcoholic served in the control group. Subjects within each of the two diagnostic groups were randomly assigned to one of three encoding instruction groups as in Experiment 1. The mean age of the Korsakoff group was 59.17 years (range: $46-68$ years), and the mean IQ was 97.66 (range: 90-106). The mean age of the alcoholic group was 55.61 years (range: $40-67$ years), and the mean IQ was 101.44 (range: 93-110).

Stimuli and Procedure. The stimulus materials and procedure were identical to those in Experiment 1 except that subjects in all groups received category label cues at the time of recall. Category labels were printed on cards and, at the time of recall, were placed one at a time in front of the subject, who was asked to recall all the stimulus words presented that belonged to that category.

\section{Results and Discussion}

The mean percentage correct recall scores for subjects in the two diagnostic groups under the three encoding instructions are shown in Figure 2. The main effect of diagnostic group was significant $[\mathrm{F}(1,30)=28.96$, $\mathrm{p}<.001]$, as was the effect of encoding instructions $[F(2,30)=42.57, p<.001]$. The interaction between these two variables was not significant $[F(2,30)<1]$.

Multiple comparisons of the recall scores for the Korsakoff subjects across the three encoding instructions showed differences between $\mathrm{S}$ and NS instructions $[F(1,30)=37.62, p<.001]$ and between NS and N instructions $[F(1,30)=31.84, p<.001]$. There were no differences between the $\mathrm{N}$ and $\mathrm{S}$ instructed groups $[F(1,30)<1]$. For subjects in the alcoholic groups, there were differences between means for the NS and $S$ instructions $[F(2,30)=30.47, p<.001]$ and between NS and $\mathrm{N}$ instructions $[\mathrm{F}(2,30)=27.87, \mathrm{p}<.001]$. There were no differences between $\mathrm{S}$ and $\mathrm{N}$ instructions $[\mathrm{F}(2,30)<1]$.

Between-group comparisons showed subjects in the 


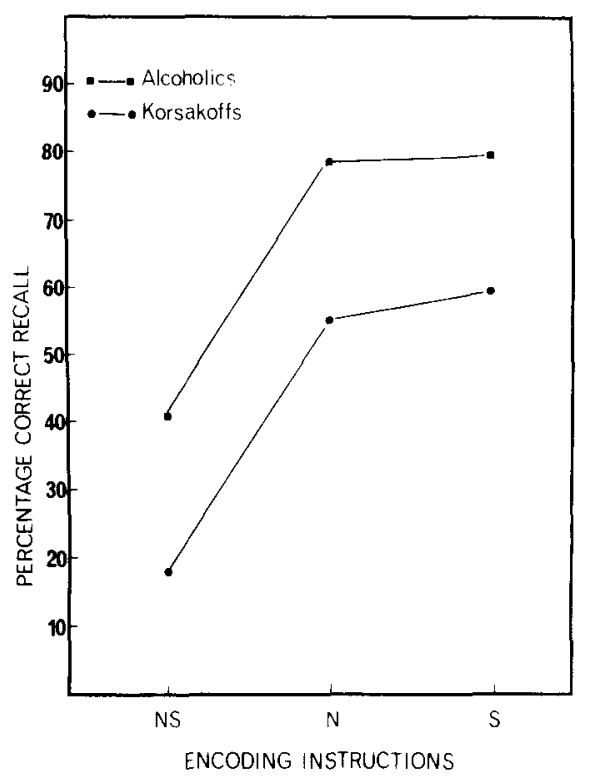

Figure 2. Mean percentage correct recall for Korsakoff and alcoholic groups in the presence of category cues at recalt, as a function of encoding instructions.

alcoholic group to have higher recall scores than Korsakoff subjects for NS instructions $[\mathrm{F}(2,30)=11.79$, $\mathrm{p}<.01]$, for $\mathrm{N}$ instructions $[\mathrm{F}(2,30)=9.40, \mathrm{p}<.01]$, and for $S$ instructions $[F(2,30)=7.96, p<.01]$.

Figure 2 shows that when Korsakoff subjects are provided with semantic cues at the time of recall, the interaction effect between diagnostic group and encoding instructions does not reach significance. Comparison of Figure 1 with Figure 2 shows that when Group $\mathrm{N}$ was provided with cues at recall time, recall scores were improved as compared with Group $\mathrm{N}$ without such cuing. In order to establish the generality of these findings while preserving internal consistency, a third experiment was designed to replicate the previous two independent ones, thus providing a direct comparison between the effects of cue vs. no cue for the three instruction groups.

\section{EXPERIMENT 3}

\section{Method}

Subjects were 72 male patients who had served in neither Experiment 1 nor Experiment 2. Thirty-six subjects had been diagnosed as alcoholic Korsakoff patients, while 36 had been diagnosed as alcoholic and served as the control group. The mean age of the Korsakoff group was 56.32 years (range: $43-67$ years), and the mean IQ was 93.00 (range: $81-110$ ). The mean age of the alcoholic group was 57.33 years (range: $39-66$ years), and the mean IQ was 96.36 (range: 88-104). Subjects within each of the two diagnostic groups were randomly assigned to one of three encoding instruction groups. Subjects within each of the groups were further divided into a group receiving category label cues at the time of recall and a group receiving no cues at the time of recall.

\section{Results and Discussion}

The mean percentage correct recall scores for the two diagnostic groups under the different encoding and cuing conditions are presented in Figure 3 . The main effect of diagnostic category was significant $[F(1,60)=47.35$, $\mathrm{p}<.001]$, as were those of encoding instructions $[F(2,60)=40.06, p<.001]$ and cuing at time of recall $[F(1,60)=27.62, p<.001]$. The Encoding by Cue interaction was significant $[F(2,60)=3.71, p<.05]$. No significant interactions were obtained for Diagnosis by Encoding $[F(2,60)=1.06]$ or Diagnosis by Cue $[F(2,60)<1)$. There was a significant Diagnosis by Encoding by Cuing interaction $[\mathrm{F}(2,60)=2.54, \mathrm{p}<.05]$.

In the absence of cues at the time of recall, Korsakoff subjects showed differences in recall between NS and S instructions $[F(1,60)=16.93, p<.001]$ and between $S$ and $\mathrm{N}$ instructions $[\mathrm{F}(1,60)=15.85, \mathrm{p}<.001]$. There were no differences in recall between $\mathrm{NS}$ and $\mathrm{N}$ instructions $[F(1,60)<1]$. When cues were provided at time of recall, Korsakoff subjects showed no differences in recall between $N$ and $S$ instructions $[F(1,60)<1]$. $\mathrm{N}$ instructions, however, resulted in higher recall performance than the NS instructions $[F(1,60)=20.36$, $p<.001]$. Analysis of the Cuing by Encoding Instruction interaction showed that cuing at the time of recall resulted in a significant increase for $\mathbf{N}$ instructions $[F(1,60)=53.29, p<.001]$ and for $S$ instructions $[F(1,60)=12.84, p<.01]$, but not for NS instructions $[F(1,60)=3.96]$. The results support the findings obtained in Experiments 1 and 2 and show that, without cuing at the time of recall, Korsakoff subjects without specific encoding instructions do not increase their recall output above the level of a group receiving instructions to encode nonsemantically. The group without explicit encoding instructions, when provided with cues at the time of recall, increased their perform. ance to a level that is similar to a group of Korsakoff subjects receiving semantic encoding instructions plus cues at recall time.



Figure 3. Mean percentage correct recall for Korsakoff and alcoholic groups as a function of encoding instructions and the presence or absence of cues at recall. 


\section{GENERAL DISCUSSION}

Cermak and Butters (1973) argued that Korsakoff patients are unable to encode stimuli semantically without specific instructions to do so. The results of Experiment 1 taken by themselves provide support for this theory, in that Korsakoff subjects receiving no explicit encoding instructions showed a recall performance that was not significantly different from a group receiving instructions to encode nonsemantically. In addition, both groups showed recall levels that were lower than a group instructed to encode semantically. The results of Experiment 2, however, do not support a semantic encoding deficit theory.

The provision of semantic cues at the time of recall should produce different results, depending on the level of encoding performed on the stimuli. According to the encoding specificity hypothesis (Tulving \& Thomson, 1973), retrieval cue effectiveness is a function of the encoding operations performed on the stimuli at the time of presentation. Stimuli encoded at a nonsemantic level should gain little benefit from semantic cues provided at recall, and evidence that this is so in normal subjects has been provided by Fisher and Craik (1977). Figure 3 shows that when semantic cues are provided at the time of recall to a group of Korsakoff subjects instructed to encode nonsemantically, no increase in recall is noted. The same semantic cues provided to a group of Korsakoff subjects instructed to encode semantically results in a significant increase in recall performance. For the group of Korsakoff subjects not provided with explicit encoding instructions, semantic cues at the time of recall increases recall performance significantly strongly to suggest that the initial encoding occurred at a semantic level, allowing semantic cues to be effective.

If Korsakoff subjects were encoding along semantic dimensions without specific instructions to do so, why, in Experiment 1, was their recall performance similar to that of the group instructed to encode nonsemantically? Alternatively, why was their recall level not similar to that of the group instructed to encode semantically? There are at least two possibilities to account for this finding. The first hypothesizes that the group receiving no encoding instructions encoded stimuli along semantic levels but to a lesser extent than the group specifically instructed to encode semantically, the higher recall performance in the latter group reflecting a deeper and more complete level of encoding. This view, however, is weakened by the results obtained in Experiment 2, in which providing cues at the time of recall increased performance in both the group receiving no encoding instructions and the group receiving semantic encoding instructions to a similar level. Presumably, if the initial level of encoding in the group receiving no explicit encoding instructions was less complete than the group instructed to semantically encode, the extent of the recall increase obtained when semantic cues were provided at recall should not have been equivalent for both groups.

The second hypothesis states that there was no difference in the levels of semantic encoding performed by the group receiving no encoding instructions and the group receiving semantic encoding instructions. The difference in recall between the two groups is accounted for by hypothesizing that for the group receiving instructions to encode semantically, category labels were presented to the subjects during the learning phase, thus providing them with direct access to superordinate retrieval cues. Subjects receiving no encoding instructions were not provided with such cues and, presumably, would have had to generate their own retrieval cuing strategy to aid recall. The low recall performance obtained by this group implies an impairment in the ability to generate such cues.

The results obtained by the alcoholic groups more nearly approximate the findings found in studies manipulating encoding instructions in normal subjects (Hyde, 1973; Hyde \& Jenkins, 1973). Thus instructions to semantically encode resulted in higher recall performance than instructions to encode nonsemantically, and a group receiving no explicit encoding instructions recalled at a level similar to a group instructed to encode semantically.

The findings of this study are not in complete agreement with those obtained by Wood and Kinsbourne (Note 1), who found with Korsakoff subjects that precuing, that is, providing the category names at the time of presentation, did not benefit recall in the absence of any postcuing, that is, providing category labels at the time of recall. Essentially, the main difference in methodology between the two studies lies in the task requirements of the subjects. In the present study, subjects receiving semantic encoding instructions were required to assign words to their appropriate superordinate labels. The finding that all Korsakoff subjects were able to do this ensured that semantic encoding was achieved. Wood and Kinsbourne (Note 1) provided subjects with the to-be-remembered words along with their appropriate category labels. There was no way of ensuring that this procedure encouraged a semantic encoding procedure or that subjects used the labels to provide a retrieval strategy.

The present study implicates an impairment in the ability of Korsakoff subjects to generate their own appropriate retrieval cues during recall, although they can use retrieval cues when they are provided. Fuld (1976) has suggested that encoding an item in such a way as to allow consistent retrieval may require that items within a list be related semantically to each other. In other words, encoding along semantic levels per se may not allow effective retrieval unless the semantic relationships among the words are perceived and encoded, for example, by linking them to some super- 
ordinate label. In the absence of specific cues at recall time, identification of superordinate labels or higher order units probably depends on perceiving semantic similarity among items. In the present study, the categorized word list was always presented with the constraint that no two members of the same category follow each other. Thus, conceptually related items were not immediately available for some form of organization. The group instructed to semantically encode in effect provided organization to the word list by linking individual items to their superordinate labels.

A related problem is the finding that Korsakoff subjects are impaired in the rate at which they process stimuli (Cermak et al., 1974; Oscar-Berman et al., 1973). Korsakoff subjects may require more time in order to sufficiently encode words such that their categorical natures are perceived. This may apply particularly when categorized word lists are presented in a nonblocked manner (i.e., when words are not grouped by category).

Finally, note should be taken of a study by Stoff and Eagle (1971), who found a positive correlation between the reported strategies adopted by normal subjects in memory tasks and their scores on the similarities subtest of the WAIS. In particular, subjects who recalled words by adopting an organizational strategy, that is, by organizing words according to semantic relatedness, had higher scores on the similarities subtest than did subjects who reported using no such organizational strategy. The authors noted that the adoption of an organizational strategy and performance on the similarities subtest have in common the examination of semantic relationships among words. These findings are related to the present study in that Korsakoff subjects typically perform very poorly on the similarities subtest. The mean scaled score on the similarities subtest for Korsakoff subjects combined across the three experiments $(\mathrm{N}=72)$ was 4.39 ; for the alcoholic subjects $(\mathrm{N}=72)$, the mean score was 7.39. The failure to organize words into their appropriate conceptual categories, which in turn may be at least partly due to an impaired rate of stimulus processing, may be related to the failure to generate retrieval cues at the time of recall.

\section{REFERENCE NOTE}

1. Wood, F., \& Kinsbourne, M. Paper in symposium on path ological forgetting, International Neuropsychological Society, February 8. 1974, Boston.

\section{REFERENCES}

Bregman, A. S. Forgetting curves with semantic, phonetic, graphic, and contiguity cues. Journal of Experimental Psychology, 1968, 78, 539-546.

CERMAK. L. S. Human memory: Research and theory. New York Ronald Press, 1972.

Cermak, L. S., \& Butters, N. The role of interference and encod ing in the short-1erm menory deticits of Korsakoff patients. Neuropsychologia. 1972, 10,89-95.

Cermak. L. S.. \& Butters, N. Information processing deficits of alcoholic Korsakoff patients. Quarterly Journal of Studies on Alcohol, 1973, 34, 1110-11.32
Cermak. L. S., Butters, N.. \& Gerrein. J. The extent of the verbal encoding ability of Korsak off patients. Neuropsychologia, $1973,11,85-94$.

Cermak, L. S.. Butters, N. \& Moreines, J. Some analyses of the verbal encoding deficit of alcoholic Korsak off patients. Brain \& Language, 1974, 1. 141-150.

CARIK, F. I. M. \& LockHART. R. S. Levels of processing: A framework for memory research. Joumal of Verbal Learning and Verbal Behavior, 1972, 11, 671-684.

Craik, F. I. M., \& Tulving, E. Depth of processing and the retention of words in episodic memory. Joumal of Experimental Psychology: General, 1975, 104. 268-294.

Fisher. R. P.. \& Craik. F. 1. M. Interaction between encoding and retrieval operations in cued recall. Joumal of Experimental Psychology: Human Learning and Memory, 1977, 3. $701-711$.

Fuı.D. P. A. Storage, retention, and retrieval in Korsakoff's syndrome. Neuropsichologia, 1976, 14, 225-236.

HY DE. T. S. Differential effects of effort and type of orienting task on recall and organization of highly associated words. Journal of Experimental Psychology. 1973, 97, 111-113.

HYDE, T. S., \& Jenkins, J, J. Recall for words as a function of semantic, graphic, and syntactic orienting tasks. Journal of Verbal Leaming and Verbul Behavior, 1973, 12, 471-480.

JAFFE. P. G.. \& Katz, A. N. Attenuating anterograde amnesia in Korsakoff's psychosis. Joumal of Abnormal Psychology, 1975. 84. 559-562.

Merssner, W. W. Learning and memory in the Korsakoff syndrome. International Journal of Neuropsychiatry, 1968, January Fibruary, 0-20.

Oscar-Berman, M. Goodglass, H., \& Cherlow, D. G. Perceptual laterality and iconic recognition of visual materials by Korsakoff patients and normal adults. Journal of Comparative and Physiological Psychology, 1973, 82, 316-321.

Peterson. L. R..\& Peterson, M. J. Short-term retention of individual verbal items. Journal of Experimental Psychology, 1959. 58. 19.3-198.

STOFF. D. M., \& EAGLE. M. N. The relationship among reported strategies. presentation rate, and verbal ability and their effects on free recall learning. Joumal of Experimental Psychology, $1471,87,423-428$.

TALI.AND, G. Psychological studies of Korsakoff's psychosis, III. Concept formation. Journal of Nervous and Mental Disease. 1459. 128. 214.226.

TAlland, G. Deranged memory. New York: Academic Press, 1965.

TIt., R. E. \& Jenkins, J. J. The effects of cued orienting tasks on tree recall of words. Journal of Verbal Learning and Verbal Bihavior, 1973, 12. 489-498.

Tulving. E.. \& Thомson, D. M. Encoding specificity and retrieval processes in episodic memory. Psychological Review, 1473. 80. 352-373.

Vicior. M.. Herman, K., \& White, E. E. A psychological study of the Wernicke-Korsak off syndrome: Results of WechslerBellevue Intelligence Scale and Wechsler Memory Scale testing at different stages in the disease. Quarterly Journal of Studies on Alcohol, 1959, 20, 467-479.

VICTOR, M., \& YAKOVLEV, P. I. S. S. Korsakoff's psychic disorder in conjunction with peripheral neuritis, a translation of Korsakoff's original article with brief comments on the author and his contribution to clinical medicine. Neurology, 1955, 5 , 394-406.

Warrington, E. K., \& Weiskrantz, L. Amnesia: Consolidation or retrieval? Nature. 1970, 228, 628-630.

Warrington, E. K., \& WeISKRantz, L. Organizational aspects of memory in anmesic patients. Neuropsychologia, 1971, 9. $67-73$.

Warrington, E. K., \& Weiskrantz, L. The effect of prior learning on subsequent retention in amnesic patients. Neuropsichologia, 1974, 12, 419-428.

Wickens, D. D. Encoding categories of words: An empirical approach to meaning. Psychological Review, 1970, 77, 1-15.

(Received for publication July 11, 1978: revision accepted February 15, 1979.) 